\title{
GLOBAL LOCAL ANALYSIS WITH ROBIN PARAMETERS: APPLICATIONS TO CRACK PROPAGATION IN 2D AND 3D MODELS
}

\section{IGNACIO FUENZALIDA-HENRIQUEZ ${ }^{1}$, EMILIO CASTILLO-IBARRA ${ }^{2}$ AND JORGE HINOJOSA ${ }^{3}$}

${ }^{1}$ Doctorado en Sistemas de Ingeniería; Engineering Building and Management Department, Universidad de Talca ifuenzalida@utalca.cl

2 Doctorado en Sistemas de Ingeniería, Universidad de Talca emcastillo@utalca.cl

${ }^{3}$ Industrial Technologies Department, Universidad de Talca jhinojosa@utalca.cl

Key words: Global, Local, Robin Parameter, Crack Propagation, Patch

\begin{abstract}
Global local analysis is a part of the structural analysis that allows to study, with an iterative solution, a coarse global linear model with a specific zone. This zone is defined as a local model with fine mesh and a non-linear behaviour such as crack propagation. However, the current trend in Global Local analysis is to impose displacements on the fine model to later obtain the reactions that will be applied to the global model for each iteration (Primal to Dual solution algorithm). Therefore, we propose a mixed analysis in the local and global models through the application of Robin conditions on the interface, allowing a higher grade of flexibility for the case of the patch or fine model with crack propagation behaviour. As a result, the algorithm converges successfully, presenting kinematic compatibility and good results with respect to the Monolithic (non-decomposed) model. Finally, a sensitivity analysis is performed on some variables regarding the crack propagation for 2D models. Finally, the proposed methodology also allows to improve the performance of the method for cracked models or other nonlinearities when compared with the current global local analysis, presented in the state of the art.
\end{abstract}

\section{INTRODUCTION}

The goal of non-intrusive frameworks is to develop advanced numerical methods benefiting from the efficient linear and non-linear solvers implemented in the commercial software, used as "black boxes". For example, it is possible to drive the non-intrusive analysis with the python interface and Code Aster [4] software. Advanced algorithms can be designed within this python interface and call non-linear methods of Code Aster to take into account complex phenomena. Depending on the point of view, the non-intrusivity would also enable to "enrich" a global model with a more detailed local one without altering the global behaviour. For instance, the global model could be the result of a long industrial design process and may not be modified easily. In that sense, methods that include local models without touching the global mesh are considered as non-intrusive. 
The global/local approach [14] is a very good candidate for non-intrusive implementation [7]. The basic idea is to introduce some localized details and potentially non-linear behaviors in a global and coarse model within specific zones and without modifying the global model. Two models coexist: a global one and a local one connected through an interface. The global/local approach consists in an iterative Dirichlet-Neumann algorithm where an iteration is composed of two steps: (1) a problem on the local model with Dirichlet boundary conditions on the interface, and (2) a problem on the global model with Neumann boundary conditions on the interface. Links with Optimized Schwarz domain decomposition methods can be found in [9]. The global/local framework has been generalized to a domain decomposition method with a complete covering local model [3]. This method has been applied on various types of non-linearities such as: crack propagation $[13,3]$, structural joints and assemblies [10], local plasticity [7], cycling visco-elastic behavior [1]. Others studies for different applications and improvements for the local global method and non-intrusive analysis can be found in $[11,6,8,9,2]$.

Based on the St. Venant principle, the interface between the local and global models should be far from the local details to avoid the Dirichlet boundary conditions from the linear global model being inaccurate near the zone of potential non-linearity. The use of Robin Parameters on the interface may help to disregard this issue. The first proposition for the use of Robin Parameters on the interface is on [6] for a local plastic model.

This paper addresses the global/local method with a non-intrusive implementation of the Robin Parameters on the interface, for the global and local models, applied to crack propagation in the fine model.

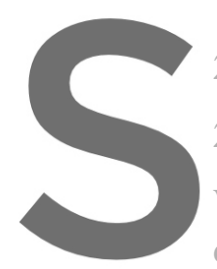

2 GLOBAL-LOCAI, PROBLEM FORMULATION
2.1 Reference problem
We consider a mechanical model of a structure defined on a doma

domain is composed of two non-overlapping domains $\Omega_{C}$ and $\Omega_{J}$.
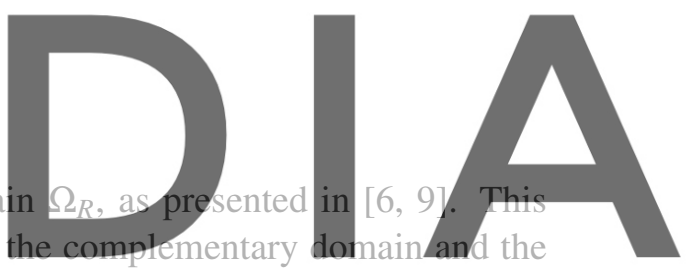

local domain, respectively. The domain $\Omega_{C}$ considers elastic linear isotropic assumptions with small

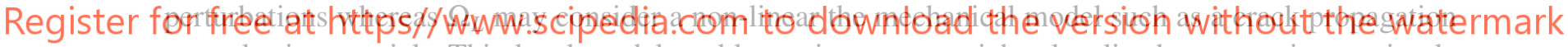
or a plastic material. This local model enables to incorporate richer localized contents into a simple global model of the structure. The interface between the complementary and the local model is defined as $\Gamma$.

The admissible space of the displacements is defined by $V\left(\Omega_{R}\right)=\left\{v \in H_{1}\left(\Omega_{R}\right), v=u_{d}\right.$ on $\left.\partial_{u} \Omega_{R}\right\}$. The mechanical problem is equivalent to:

$$
\text { Find } u \in V\left(\Omega_{R}\right), a_{R}(u, v)=l_{R}(v), \forall v \in V_{0}\left(\Omega_{R}\right)
$$

The bi-linear form representing the equilibrium of the structure is defined with $a_{R}$ and the linear form representing the loads and the boundary conditions with $l_{R}$.

A standard finite element discretization with Lagrange shape functions is used to obtain discrete models and we assume a conforming mesh at the interface between the complementary and local models.

In order to detail the methods and the equation, we adopt a full elastic linear isotropic material over the whole domain $\Omega_{R}=\Omega_{C} \cup \Omega_{L}$. The case of a local non-linear model will be discussed further. Under this 
type of hypothesis, the discrete problem becomes:

$$
\text { Find } \mathbf{u}_{R} \text { solution of } \mathbf{K}_{R} \mathbf{u}_{R}=\mathbf{f}_{d}^{R}
$$

the stiffness matrix is defined with $\mathbf{K}_{R}$, the discrete unknown of displacements with $\mathbf{u}_{R}$ and the righthand-side corresponding to the Boundary conditions with $\mathbf{f}_{d}^{R}$. The null Dirichlet conditions are supposed to be eliminated.

This reference problem can be observed as the coupling between the complementary model on $\Omega_{C}$ and the local model on $\Omega_{L}$. This coupling is enforced through a Lagrangian $\lambda$ to impose the continuity of the displacement on the interface $\Gamma$. Therefore, the discrete problem is equivalent to:

$$
\text { Find }\left(\mathbf{u}_{C}, \mathbf{u}_{L}, \boldsymbol{\lambda}\right),\left[\begin{array}{ccc}
\mathbf{K}_{C} & 0 & -\mathbf{C}_{C}^{T} \\
0 & \mathbf{K}_{L} & \mathbf{C}_{L}^{T} \\
-C_{C} & C_{L} & 0
\end{array}\right]\left[\begin{array}{c}
\mathbf{u}_{C} \\
\mathbf{u}_{L} \\
\lambda
\end{array}\right]=\left[\begin{array}{c}
\mathbf{f}_{d}^{C} \\
\mathbf{f}_{d}^{L} \\
0
\end{array}\right]
$$

the stiffness matrices of the complementary and local models are defined with $\mathbf{K}_{C}$ and $\mathbf{K}_{L}$, respectively, the displacements on $\Omega_{C}$ and $\Omega_{L}$ with $\mathbf{u}_{C}$ and $\mathbf{u}_{L}$, the load vectors on $\Omega_{C}$ and $\Omega_{L}$ with $\mathbf{f}_{d}^{C}$ and $\mathbf{f}_{d}^{L}$, and the Lagrange's multiplier to enforce the coupling with $\lambda$.

The operators $\mathbf{C}_{C}$ and $\mathbf{C}_{L}$ are coupling operators. In the case of conforming meshes, these are trace operators extracting the displacements from $\Omega_{C}$ and $\Omega_{L}$ on the interface $\Gamma$. They are sparse matrices with 1 in the degrees of freedom of the interface.

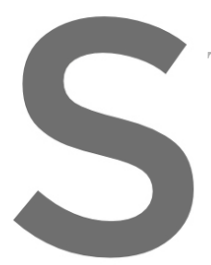

This problem can be re
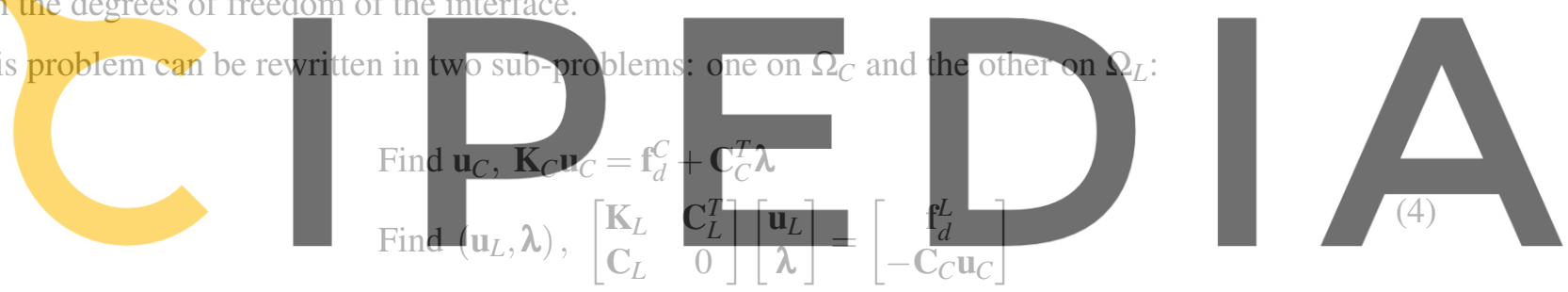

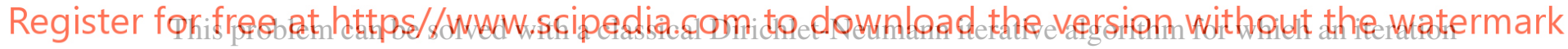
consists of the two following steps:

1. Knowing a solution $\left(\mathbf{u}_{L}^{n}, \lambda^{n}\right)$ on $\Omega_{L}$, solve a Neumann problem on $\Omega_{C}$ :

$$
\text { Find } \mathbf{u}_{C}^{n+1}, \mathbf{K}_{C} \mathbf{u}_{C}^{n+1}=\mathbf{f}_{d}^{C}+\mathbf{C}_{C}^{T} \lambda^{n}
$$

2. Knowing a solution $\mathbf{u}_{C}^{n+1}$ on $\Omega_{C}$, solve a Dirichlet problem on $\Omega_{L}$ :

$$
\text { Find }\left(\mathbf{u}_{L}^{n+1}, \lambda^{n+1}\right),\left[\begin{array}{cc}
\mathbf{K}_{L} & \mathbf{C}_{L}^{T} \\
\mathbf{C}_{L} & 0
\end{array}\right]\left[\begin{array}{c}
\mathbf{u}_{L}^{n+1} \\
\lambda^{n+1}
\end{array}\right]=\left[\begin{array}{c}
\mathbf{f}_{d}^{L} \\
\mathbf{C}_{C} \mathbf{u}_{C}^{n+1}
\end{array}\right]
$$

$\lambda^{n+1}$ the opposite of the reaction forces $\lambda_{L}^{n+1}$ on the interface of the local model.

This type of algorithm could be generalized with multiple local models and considered as a non-overlapping domain decomposition [3]. 


\section{GLOBAL-LOCAL ANALYSIS WITH ROBIN PARAMETERS}

As stated before, the global/local analysis can be observed as a coupling of the complementary and the local model through a Lagrange's multiplier. This coupling problem is solved with an iterative DirichletNeumann fixed point algorithm [9].

However, similarly as for Schwarz domain decomposition methods [5], we can introduce Robin conditions to improve the convergence and obtain more flexibility. In fact, the kinematic compatibility is loss between the complementary and local model and replace it with Robin conditions written on the interface $\Gamma$. The first work on the global/local method with Robin conditions are presented in [6] and reminded in [9]. In Section 3.1 the complete derivation global/local method with Robin conditions is presented.

\subsection{Derivation of global/local Analysis with Robin Parameters}

In this section, the mechanical problem is written differently following a framework of a mixed domain decomposition. Instead of considering a Lagrange's multiplier defined on the interface $\Gamma$, we consider the reaction forces $\lambda_{L}$ and $\lambda_{C}$ as full unknowns on the interface. Writing the problem as a domain decomposition enables the conditions to enforce at the interface:

\section{$\lambda_{L}+\lambda_{C}=0 \quad$ Equilibrium of the forces \\ $\mathbf{C}_{L} \mathbf{u}_{L}-\mathbf{C}_{C} \mathbf{u}_{C}=0 \quad$ Continuity of the displacements}

\section{These two equations representing the behavior of the interface are written with Robin conditions:}
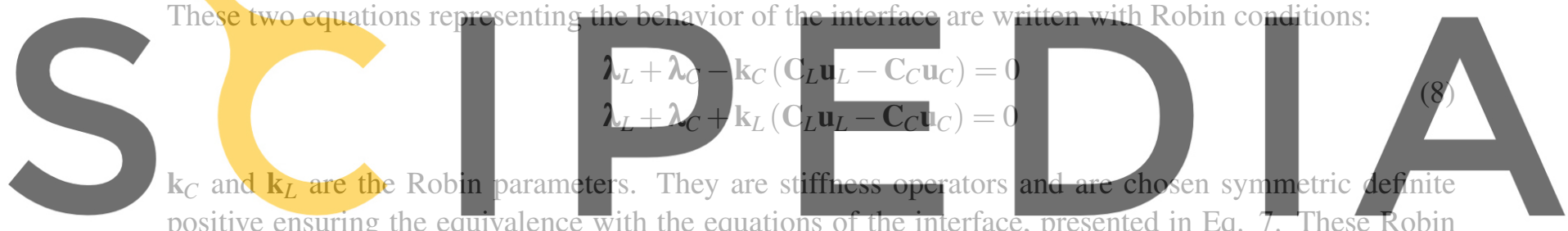

positive ensuring the equivalence with the equations of the interface, presented in Eq.

conditions are defined and written on the interfaces: they connect the reaction forces $\lambda_{C}$ and $\lambda_{L}$ to the

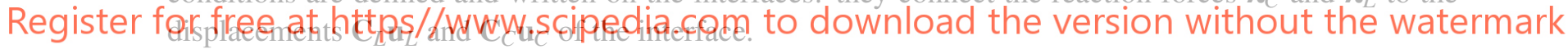

Therefore, the new system to be solved is:

$$
\begin{aligned}
& \mathbf{K}_{C} \mathbf{u}_{C}=\mathbf{f}_{d}^{C}+\mathbf{C}_{C}^{T} \boldsymbol{\lambda}_{C} \\
& \boldsymbol{\lambda}_{L}+\boldsymbol{\lambda}_{C}-\mathbf{k}_{C}\left(\mathbf{C}_{L} \mathbf{u}_{L}-\mathbf{C}_{C} \mathbf{u}_{C}\right)=0 \\
& \mathbf{K}_{L} \mathbf{u}_{L}=\mathbf{f}_{d}^{L}+\mathbf{C}_{L}^{T} \boldsymbol{\lambda}_{L} \\
& \boldsymbol{\lambda}_{L}+\boldsymbol{\lambda}_{C}+\mathbf{k}_{L}\left(\mathbf{C}_{L} \mathbf{u}_{L}-\mathbf{C}_{C} \mathbf{u}_{C}\right)=0
\end{aligned}
$$

As well as the Dirichlet-Neumann global/local method, the problem on the complementary model is extended to the global model and the first equation of the system becomes:

$$
\mathbf{K}_{G} \mathbf{u}_{G}=\mathbf{f}_{d}^{G}+\mathbf{C}_{G}^{T} \boldsymbol{\lambda}_{C}+\mathbf{C}_{G}^{T} \boldsymbol{\lambda}_{A}
$$

In addition, as $\mathbf{u}_{G}$ and $\mathbf{u}_{C}$ are equal on the interface $\Gamma$, the Robin conditions can also be written as:

$$
\begin{aligned}
& \boldsymbol{\lambda}_{L}+\boldsymbol{\lambda}_{C}-\mathbf{k}_{G}\left(\mathbf{C}_{L} \mathbf{u}_{L}-\mathbf{C}_{G} \mathbf{u}_{G}\right)=0 \\
& \boldsymbol{\lambda}_{L}+\boldsymbol{\lambda}_{C}+\mathbf{k}_{L}\left(\mathbf{C}_{L} \mathbf{u}_{L}-\mathbf{C}_{G} \mathbf{u}_{G}\right)=0
\end{aligned}
$$


On the other hand, $\boldsymbol{\lambda}_{C}$ are the reaction forces of a problem on the complementary domain. Therefore, the notation $\boldsymbol{\lambda}_{G}$ will not be used, in order to avoid any confusion regarding $\boldsymbol{\lambda}_{G}$.

Considering the first equation of the Robin conditions, we extract the expression of $\boldsymbol{\lambda}_{C}$ to inject it into the equilibrium of the global model leading to:

$$
\mathbf{K}_{G}+\mathbf{u}_{G}=\mathbf{f}_{d}^{G}+\mathbf{C}_{G}^{T} \boldsymbol{\lambda}_{A}-\mathbf{C}_{G}^{T}\left(\boldsymbol{\lambda}_{L}-\mathbf{k}_{G} \mathbf{C}_{G} \mathbf{u}_{L}-\mathbf{k}_{G} \mathbf{C}_{G} \mathbf{u}_{G}\right)
$$

Using the second equation of the Robin conditions, the expression of $\lambda_{L}$ can be extracted to be injected it into the equilibrium of the local model obtaining:

$$
\left(\mathbf{K}_{L}+\mathbf{C}_{L}^{T} \mathbf{k}_{L} \mathbf{C}_{L}\right) \mathbf{u}_{L}=\mathbf{f}_{d}^{L}+\mathbf{C}_{L}^{T}\left(\mathbf{k}_{L} \mathbf{C}_{G} \mathbf{u}_{G}-\boldsymbol{\lambda}_{C}\right)
$$

As well as the global/local method, a fixed point algorithm is derived, where an iteration consists of the successive steps:

1. A global problem: knowing $\left(\mathbf{u}_{L}^{n}, \lambda_{L}^{n}, \lambda_{A}^{n}\right)$, find $\mathbf{u}_{G}^{n+1}$ solution of:

$$
\mathbf{K}_{G} \mathbf{u}_{G}^{n+1}=\mathbf{f}_{d}^{G}+\mathbf{C}_{G}^{T} \underbrace{\left(\lambda_{A}^{n}-\left(\lambda_{L}^{n}-\mathbf{k}_{G} \mathbf{C}_{L} \mathbf{u}_{L}^{n}-\mathbf{k}_{G} \mathbf{C}_{G} \mathbf{u}_{G}^{n}\right)\right)}_{\mathbf{P}^{n}}
$$

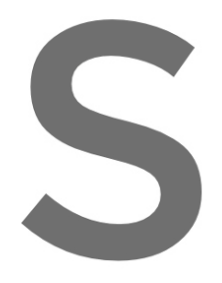

2. Auxiliary problems: computing the reaction forces on $\Omega_{A}$ and $\Omega_{C}$ at the interface $\Gamma$ :

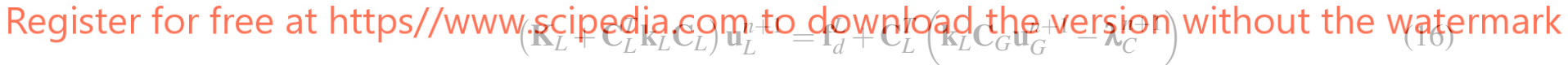

In order to save the global model without any changes, the contribution of $\mathbf{u}_{G}$ due to the Robin parameters $\mathbf{k}_{G}$ is moved to the right-hand side of the equation, with the term $-\mathbf{C}_{G}^{T} \mathbf{k}_{G} \mathbf{C}_{G} \mathbf{u}_{G}^{n}$.

For global local analysis, auxiliary problems are not necessary if the finite element codes can extract reaction forces on an immersed surface. Nevertheless, it can be computed from known variables without assembling the operator $\mathbf{K}_{C}$ :

$$
\begin{aligned}
\lambda_{C}^{n+1} & =\mathbf{C}_{C}\left(\mathbf{K}_{C} \mathbf{u}_{G}^{n+1} \Omega_{C}-\mathbf{f}_{d}^{C}\right) \\
& =\mathbf{C}_{G}\left(\mathbf{K}_{G} \mathbf{u}_{G}^{n+1}-\mathbf{f}_{d}^{G}-\left(\overline{\mathbf{K}}_{A} \mathbf{u}_{G}^{n+1}-\overline{\mathbf{f}}_{d}^{A}\right)\right) \\
& =\mathbf{C}_{G}\left(\mathbf{C}_{G}^{T} \mathbf{P}^{n}-\boldsymbol{\lambda}_{A}^{n+1}\right) \\
\lambda_{C}^{n+1} & =\mathbf{P}^{n}-\boldsymbol{\lambda}_{A}^{n+1}
\end{aligned}
$$


In addition, since $\mathbf{P}^{n}=\lambda_{A}^{n}-\lambda_{L}^{n}+\mathbf{k}_{G} \mathbf{C}_{L} \mathbf{u}_{L}^{n}-\mathbf{k}_{G} \mathbf{C}_{G} \mathbf{u}_{G}^{n}$, we can write $\mathbf{P}^{n+1}$ in function of $\mathbf{P}^{n}$ and forming a new rest $\mathbf{r}^{n+1}$ :

$$
\begin{aligned}
\mathbf{P}^{n+1} & =\boldsymbol{\lambda}_{A}^{n+1}-\lambda_{L}^{n+1}+\mathbf{k}_{G} \mathbf{C}_{L} \mathbf{u}_{L}^{n+1}-\mathbf{k}_{G} \mathbf{C}_{G} \mathbf{u}_{G}^{n+1} \\
& =\mathbf{P}^{n}-\lambda_{C}^{n+1}-\lambda_{L}^{n+1}+\mathbf{k}_{G} \mathbf{C}_{L} \mathbf{u}_{L}^{n+1}-\mathbf{k}_{G} \mathbf{C}_{G} \mathbf{u}_{G}^{n+1} \\
& =\mathbf{P}^{n}-\left[\left(\lambda_{C}^{n+1}+\lambda_{L}^{n+1}\right)+\mathbf{k}_{G}\left(\mathbf{C}_{G} \mathbf{u}_{G}^{n+1}-\mathbf{C}_{L} \mathbf{u}_{L}^{n+1}\right)\right] \\
& =\mathbf{P}^{n}+\mathbf{r}^{n+1}
\end{aligned}
$$

The new rest $\mathbf{r}^{n+1}$ now includes a mixed contribution of the discontinuity of the displacements and the disequilibrium of forces:

$$
\mathbf{r}^{n+1}=-\underbrace{\left(\lambda_{C}^{n+1}+\lambda_{L}^{n+1}\right)}_{\text {Disequilibrium }}-\mathbf{k}_{G} \underbrace{\left(\mathbf{C}_{G} \mathbf{u}_{G}^{n+1}-\mathbf{C}_{L} \mathbf{u}_{L}^{n+1}\right)}_{\text {Discontinuity }}
$$

In addition, the interface error measured as the norm of the difference of displacements between the local and global models is used to measure the quality of the solution. The expression is presented in Eq. (20).

$$
\eta_{\Gamma}=\frac{\left\|\left(\mathbf{C}_{L} \mathbf{u}_{L}^{n+1}-\mathbf{C}_{G} \mathbf{u}_{G}^{n+1}\right)\right\|_{2}}{\left\|\mathbf{C}_{G} \mathbf{u}_{G}^{n+1}\right\|_{2}}
$$

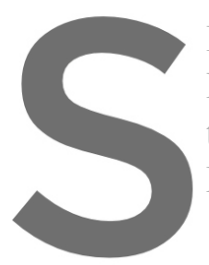

It is also relevant to $\mathrm{c}$

Robin parameters with the

the displacements on the

Monolithic model, as pr
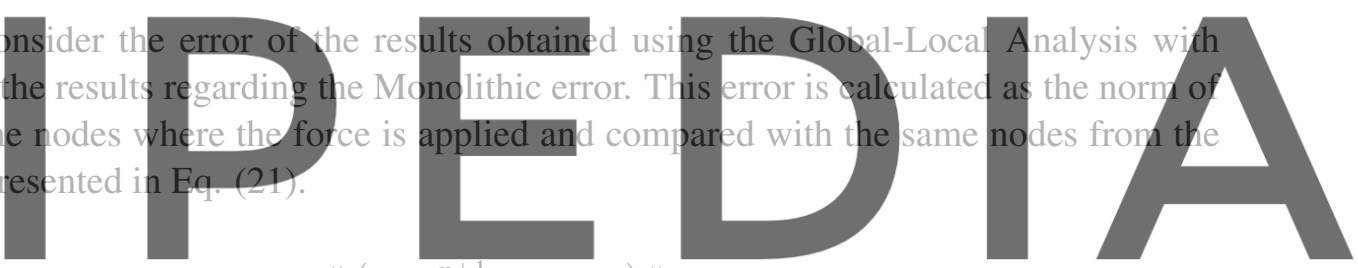

$\frac{\left\|\left(\mathbf{C}_{G} \mathbf{u}_{G}^{n+1}-\mathbf{u}_{\text {Mono. }}\right)\right\|_{2}}{2}$

(21)

Register for free at https//www.scipedia.com to down load the version without the watermark

4 RESULTS FOR 2D STRUCTURES USING GLOBAL-LOCAL ANALYSIS WITH ROBIN PARAMETERS

The Global-Local algorithm with Robin Parameters was implemented with Code Aster [4], considering two A-36 steel structures modeled with 2D plane stress formulation and a thickness equal to 1 . The structures are shaped as an inverted T-Shape with a $10 \mathrm{~mm}$ initial crack and considering different load conditions. The corresponding properties are presented in Fig. 1 and Table 1: 

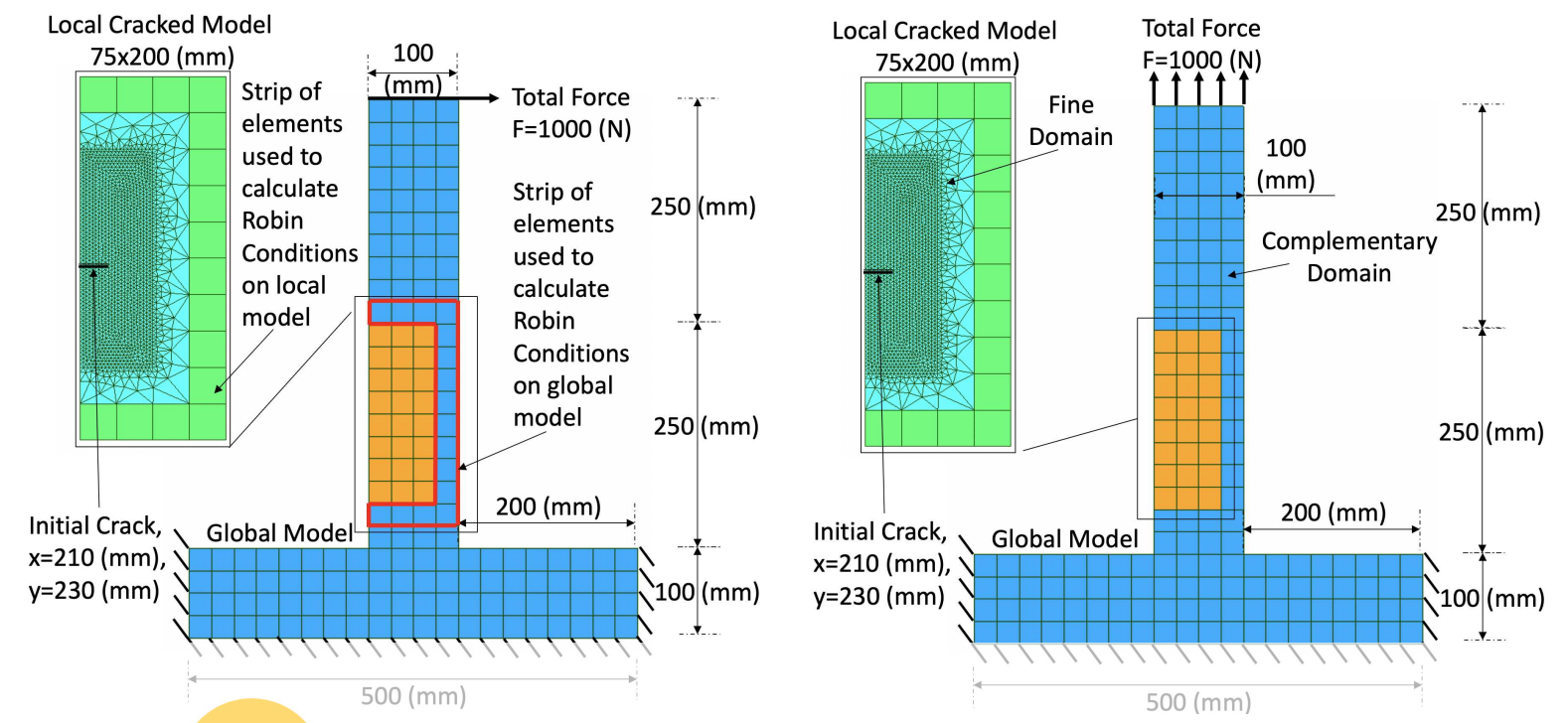

(a) Inverted T-Shaped cracked problem, Horizontal Force (b) Inverted T-Shaped cracked problem, Vertical Force Figure 1: Geometry of analyzed models, boundary conditions and applied loads
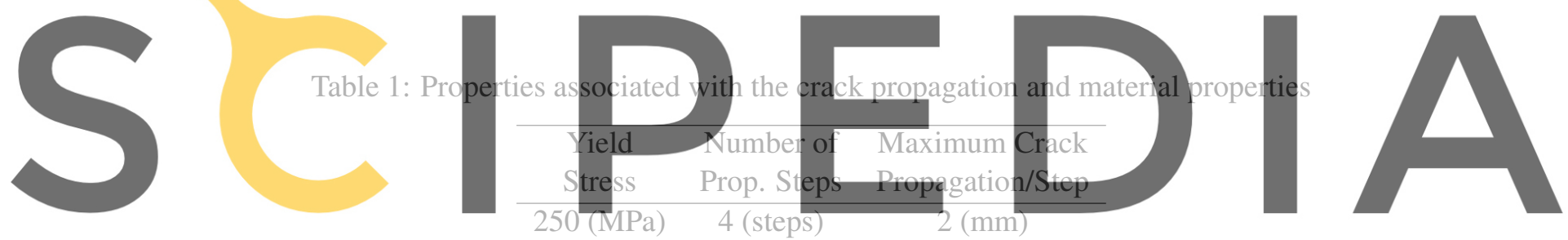

Register for free at https//www.scipedia.com to download the version without the watermark In order to analyze the performance of the Global-Local algorithm with Robin Parameters, the predefined element strip is used to calculate the Robin parameter, presented in Fig. 1b, as well as the Aitken $\delta^{2}$ dynamic relaxation, as presented in [9].

The results for the horizontal applied load and the 4 propagation steps are presented in Fig. 2 and Fig. 3. 


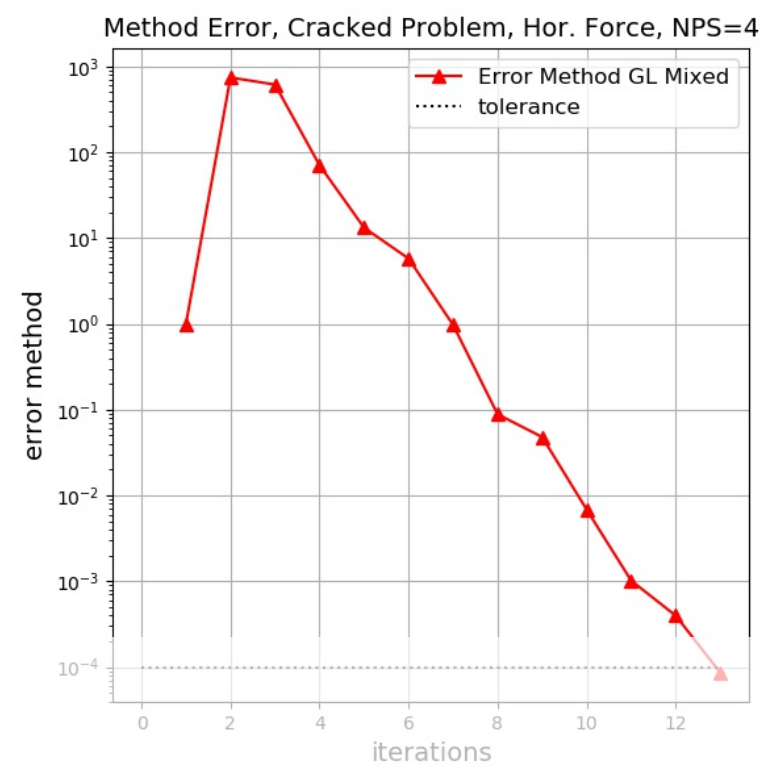

Figure 2: Evolution of Method error r/ iterations, horizontal load

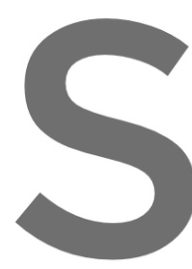

Error r/ interface, Cracked Problem, Hor. Force, NPS=4
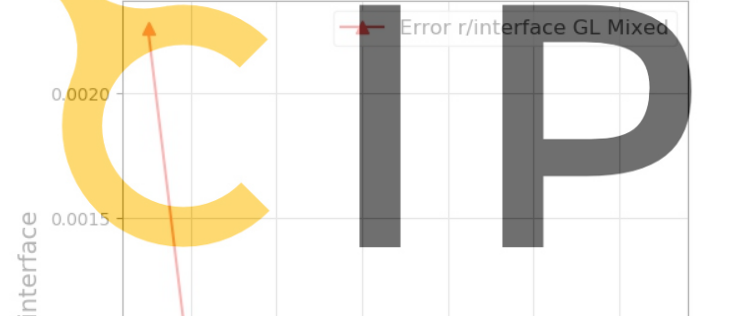

Error r/ Monolithic, Cracked Problem, Hor. Force, NPS=4
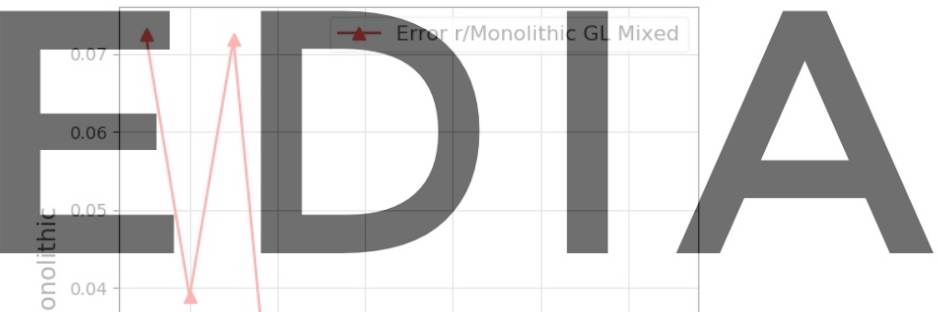

Register fợ free at https//www.scipedia.com to dō whnload the version without the watermark

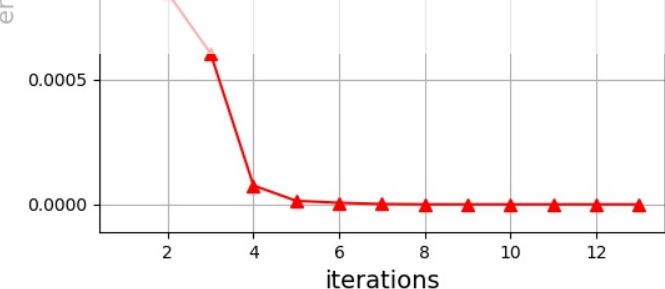

(a) Interface error r/iterations, horizontal load

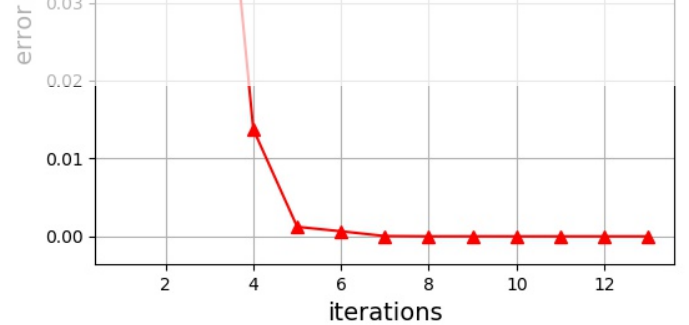

(b) Monolithic error r/iterations, horizontal load

Figure 3: Interface error and Monolithic error, horizontal load

As presented in Fig. 3a and Fig. 3b, the convergence is fulfilled in 13 iterations for the horizontal load. For the final converged state, the interface and monolithic errors tend to 0 , indicating kinematic 
compatibility between models and a good quality of the solution, respectively.

The results for the vertical applied load and the 4 propagation steps are presented in Fig. 4 and Fig. 5.
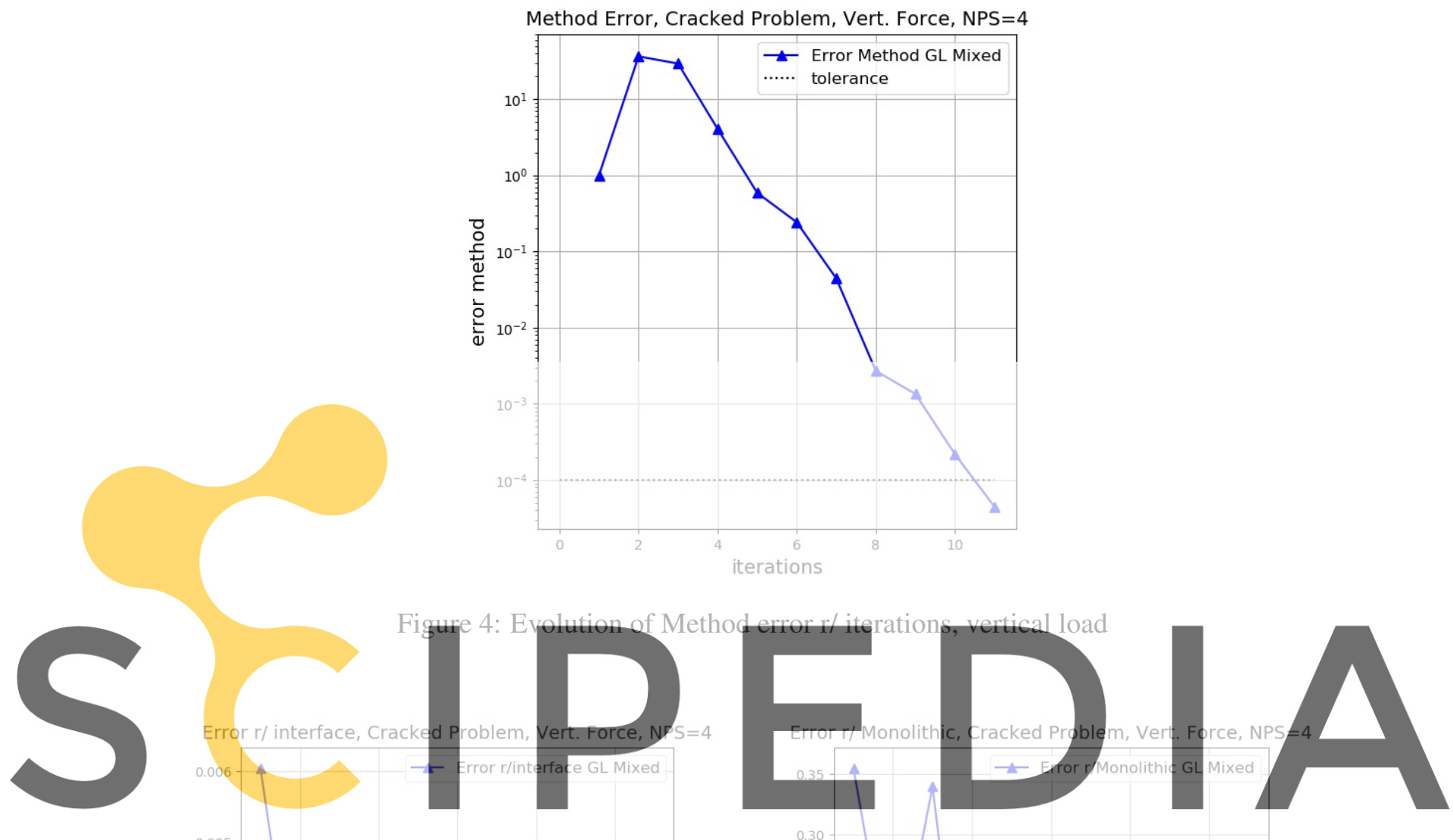

Register for free at https//www.scipedia.com to download the version without the watermark

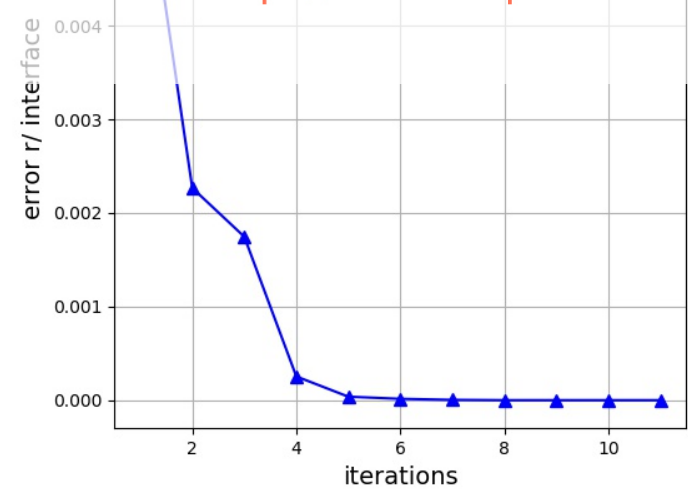

(a) Interface error r/iterations, vertical load

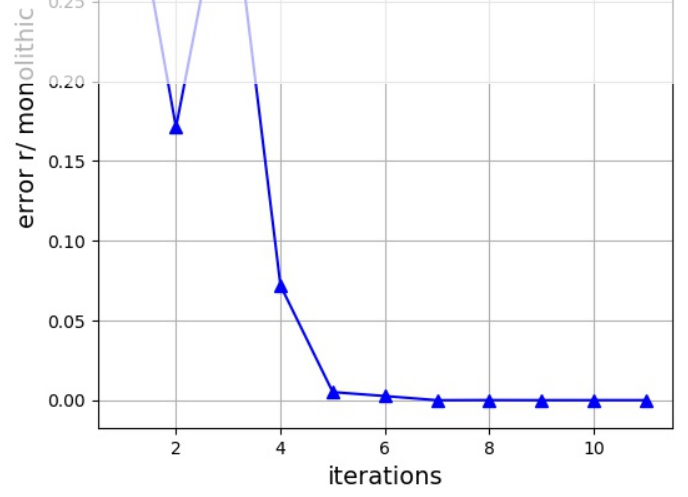

(b) Monolithic error r/iterations, vertical load

Figure 5: Interface error and Monolithic error, vertical load 
As presented in Fig. 5a and Fig. 5b, the convergence is fulfilled in 11 iterations for the horizontal load. For the final converged state, the interface and monolithic errors tend to 0 , indicating kinematic compatibility between models and a good quality of the solution, respectively.

In addition, the Aitken $\delta^{2}$ is appropriate for the Global Local Analysis with Robin Conditions, exhibiting a good convergence rate. This is presented in the slope of the method error r/iterations in Fig. 2 and Fig. 4.

Finally, as stated in [12], the convergence of the method $\left\|r^{n+1}\right\|<t o l=10^{-4}$, depends directly on the Robin parameter chosen for the analysis. Therefore, the presented convergence can be improved, due to the arbitrary strip of elements chosen in the current example and used for the calculation of the Robin parameter.

The complete results of the Global-Local Analysis with the initial Robin Operator are presented in Table 2 .

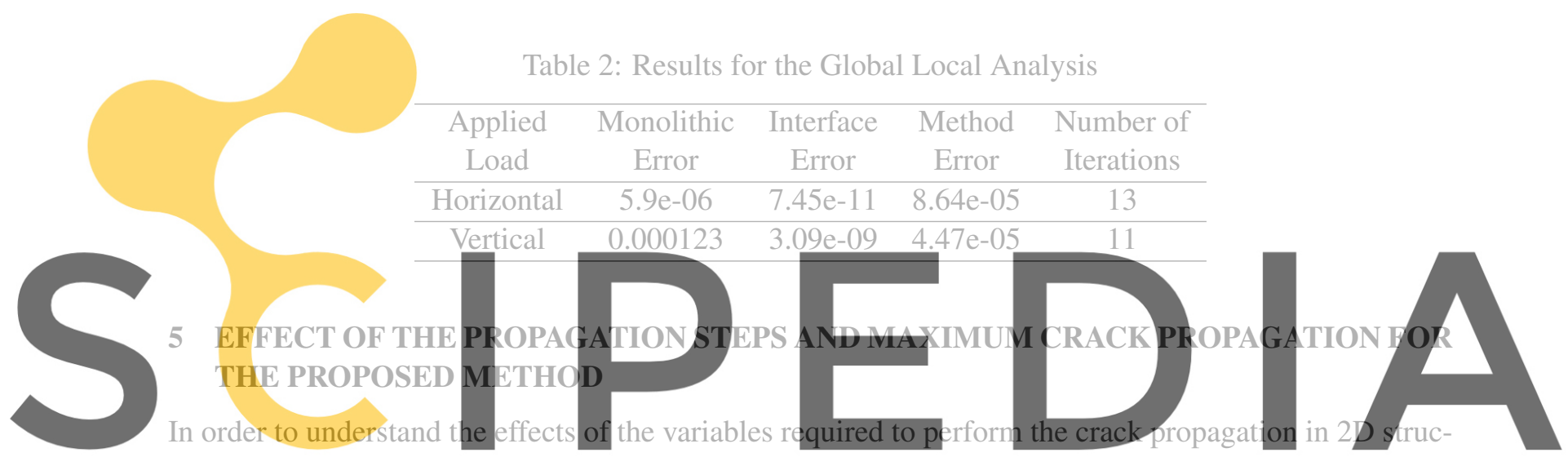

tures and how they affect the convergence rate of the Global-Local analysis with Robin parameters, the

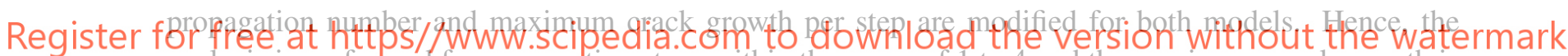
analysis is performed for propagation steps within the range of 1 to 4 and the maximum crack growth is chosen from 2 ( $\mathrm{mm})$ to 10 ( $\mathrm{mm})$ for both forces applied.

The results for the different number of propagation steps and a maximum crack growth of $2(\mathrm{~mm})$ are presented in Fig. 6a and Fig. 6b for the horizontal load with $2(\mathrm{~mm})$ and $10(\mathrm{~mm})$ crack growth (DA), respectively. It is important to mention that as presented in Section 4, the interface and monolithic errors tends to 0 ; therefore, they will be omitted in the current section. 


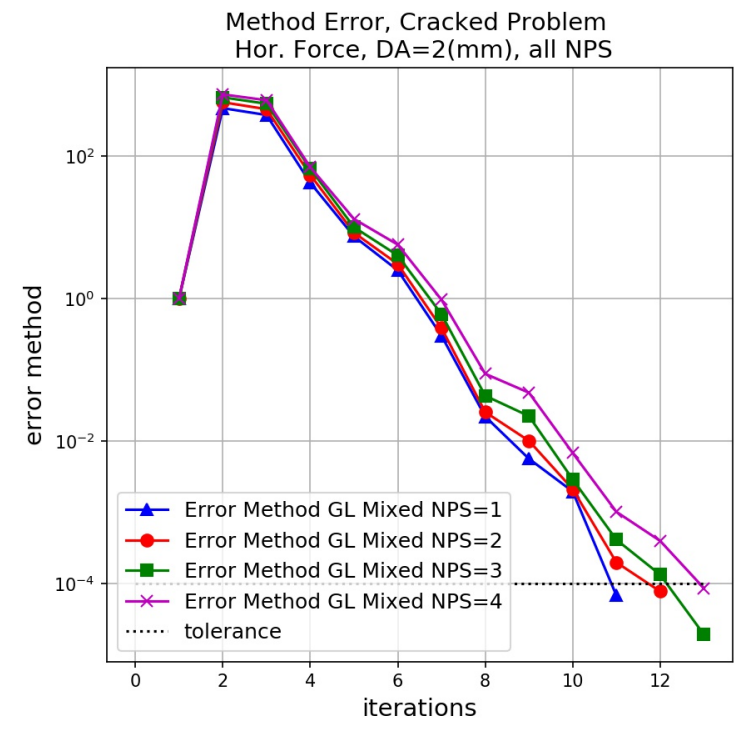

(a) Method error for all NPS, DA=2(mm), horiz. load

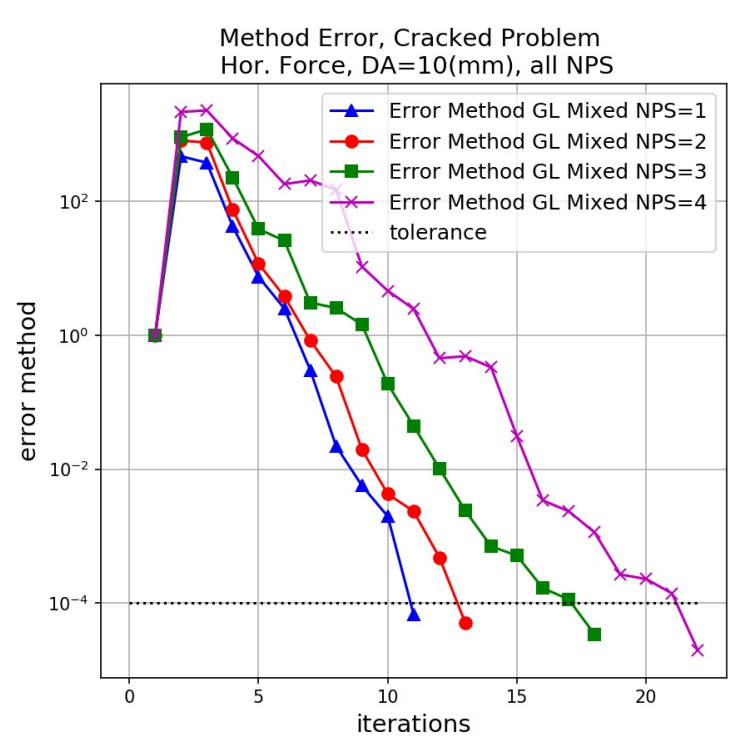

(b) Method error for all NPS, DA=10(mm), horiz. load

Figure 6: Effect of number of propagation steps for horizontal loads and different crack growth

It can be observed that the number of propagation steps analyzed affects the convergence rate of the proposed method. For the horizontal load and $\mathrm{DA}=2(\mathrm{~mm})$, the convergence is achieved with 11 iterations for the initialized crack (NPS=1), 12 iterations for one propagation (NPS=2) and 13 iterations for NPS=3 and NPS $=4$. However, when a larger crack growth is considered with $\mathrm{DA}=10(\mathrm{~mm})$, the convergence is equal to 11 for NPS=1, 13 iterations for NPS=2, 18 iterations for NPS=3 and 22 iterations for NPS=4. These results show that for a large crack growth, the discontinuity becomes geometrically larger with more propagation steps. Consequently, as to achieve a good stress and displacement compatibility, more iterations are required to fulfill the convergence.

For the vertical load case, the results for $2(\mathrm{~mm})$ and $10(\mathrm{~mm})$ crack growth and all number of propagation steps are presented in Fig. 7a and Fig. 7b. 


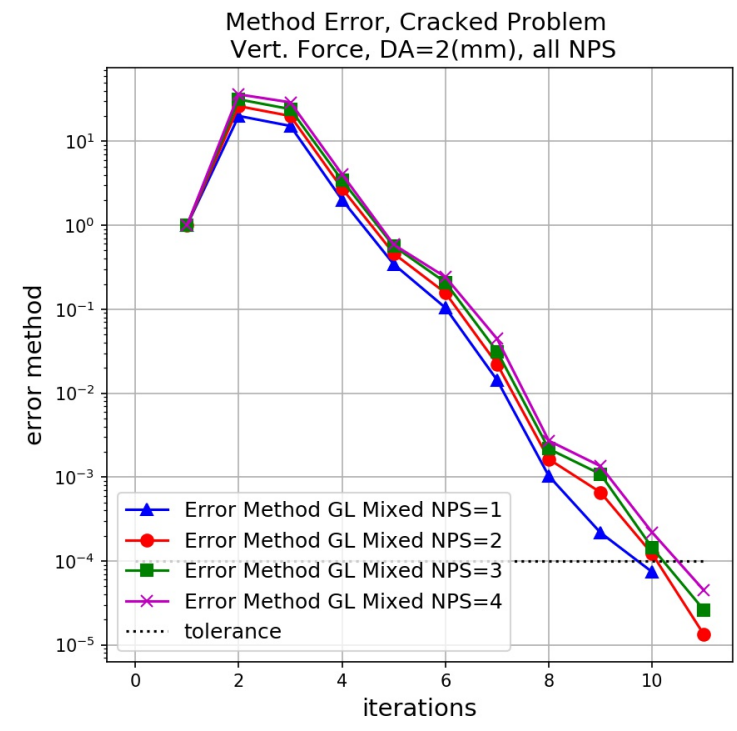

(a) Method error for all NPS, DA=2(mm), vert. load

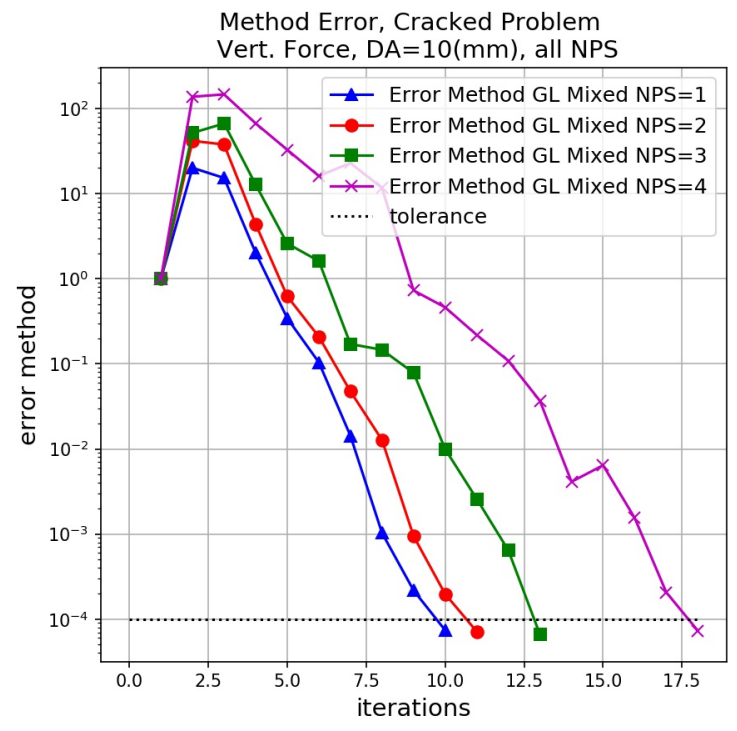

(b) Method error for all NPS, DA=10(mm), vert. load

Figure 7: Effect of number of propagation steps for vertical loads and different crack growth

As for the vertical loads, it can be observed that the number of propagation steps analyzed also affects the convergence rate of the proposed method. For the vertical load and DA=2 (mm), the convergence is achieved with 10 iterations for the initialized crack $(\mathrm{NPS}=1)$ and 11 iterations for all other cases. Nevertheless, when a larger crack growth is considered with $D A=10(\mathrm{~mm})$, the convergence is equal to 10 for NPS $=1,11$ iterations for NPS=2, 13 iterations for NPS $=3$ and 18 iterations for NPS $=4$. These results are aligned with the previous results for the horizontal load, indicating that the size of the discontinuity affects the number of iterations required to achieve a "balanced" interface condition and thus, obtaining the desired convergence.

Finally, the final deformed shape of the cracked model with 4 propagation steps and different crack growth is presented in Fig. 8a and Fig. 8b, for the horizontal loads. 


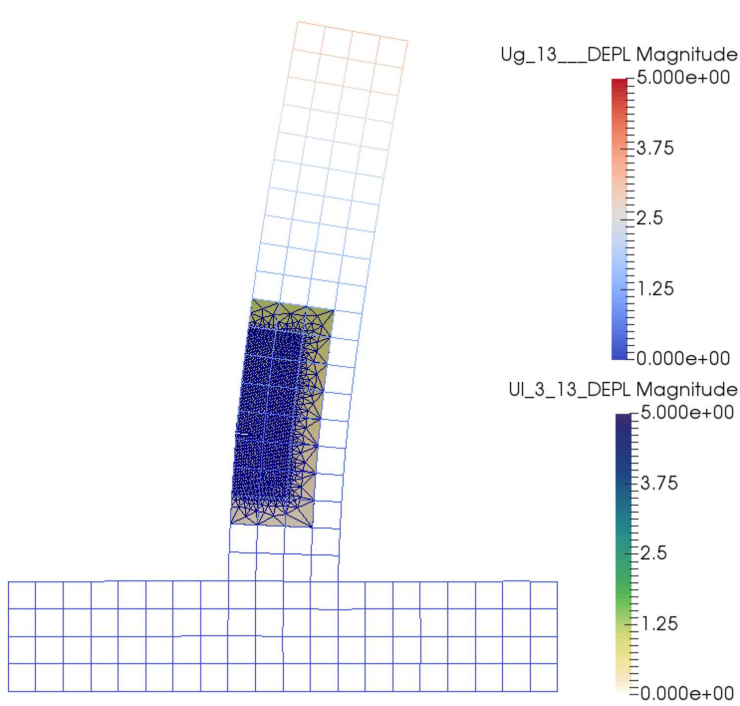

(a) Deformed state, NPS=4, DA=2(mm), horiz. load

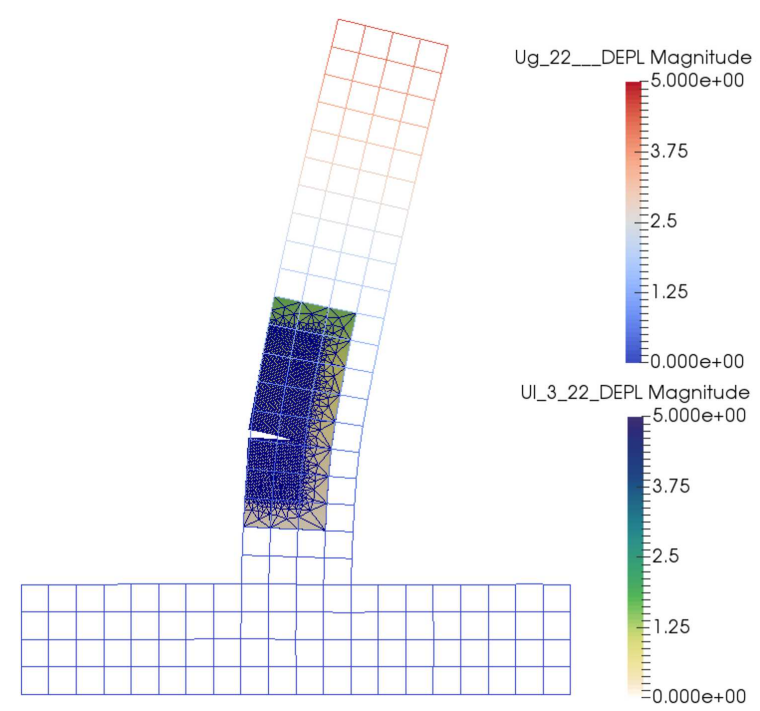

(b) Deformed state, NPS=4, DA=10(mm), horiz. load

Figure 8: Final deformed state with 4 propagation steps and $\mathrm{DA}=2(\mathrm{~mm})$ and $10(\mathrm{~mm})$ and horizontal load.

\section{CONCLUSIONS}

- The method presented in this study has a good performance regarding the resolution of structural $/$ mechanical problems with crack propagation in $2 \mathrm{D}$ plane stress elements. However, the implementation is more complex regarding the standard Global Local analysis (Primal-Dual method, presented in [9]).

- An advantage of the proposed model corresponds to the Robin Operator and the flexibility that is added to the local fine model, which can be optimized in order to improve the quality of the solution.

- The solution obtained with the Global-Local with Robin Conditions is affected by the crack growth parameter (DA) and the number of propagation steps (NPS), obtaining a best convergence rate for lower crack growth and lower number of propagation steps. These can be explained because the behaviour acts more like the original structure and, in consequence, obtaining good results for the number of iterations until convergence.

- Finally, the global-local analysis with Robin parameters presents a good correlation to the results obtained with the Monolithic solver, assuring that the analysis can be used without losing information or quality of the solution for larger models. 


\section{FUTURE RESEARCH AND COMMENTARY}

- As presented in the conclusions, the Robin parameter can be optimized in order to achieve a better convergence of the method. Basin-Hopping Optimization Algorithm can be used to find a global minimum or, at least, a better solution than the current one presented in this article.

- The extension of the method to 3D structures has been studied using the proposed method, but the results were not included in this article due to great computational effort involved.

- The proposed method can be used to solve a non-linear material, such as isotropic hardening and other types of localized non-linearities. These behaviours will be addressed in future works.

\section{ACKNOWLEDGEMENTS}

The authors acknowledges the assistance of Dr. Paul Oumaziz of Institut Clément Ader (ICA), Université de Toulouse, for the revision of the code aster routines needed for the preparation of this article. Also, the authors acknowledges the financial support from ANID PFCHA / DOCTORADO BECAS CHILE / 2018 - 21181707 and BECA ESTUDIO DE DOCTORADO UNIVERSIDAD DE TALCA.

\section{REFERENCES}

[1] Blanchard, M., Allix, O., Gosselet, P., Desmeure, G.: Space/time global/local noninvasive coupling strategy: Application to viscoplastic structures. Finite Elements in Analysis and Design 156, 1-12 (2019). DOI 10.1016/j.finel.2019.01.003

[2] Bouclier, R., Passieux, J.C., Salaün, M.: Local enrichment of NURBS patches using a non-intrusive coupling strategy: Geometric details, local refinement, inclusion, fracture. Computer Methods in Applied Mechanics and Engineering 300, 1-26 (2016). DOI 10.1016/j.cma.2015.11.007

[3] Duval, M., Passieux, J.c., Salaün, M., Guinard, S.: Non-intrusive Coupling: Recent Advances and Scalable Nonlinear Domain Decomposition. Archives of Computational Methods in Engineering 23(1), 17-38 (2016). DOI 10.1007/s11831-014-9132-x

[4] EDF: Code Aster, Analysis of Structures and Thermomechanics for Studies and Research (2019). URL www. code-aster.org

[5] Gander, M.J.: Optimized Schwarz Methods. SIAM Journal on Numerical Analysis 44(2), 699731 (2006). DOI 10.1137/S0036142903425409. URL http://epubs.siam.org/doi/10.1137/ S0036142903425409

[6] Gendre, L., Allix, O., Gosselet, P.: A two-scale approximation of the Schur complement and its use for non-intrusive coupling. International Journal for Numerical Methods in Engineering 87(9), 889-905 (2011). DOI 10.1002/nme.3142

[7] Gendre, L., Allix, O., Gosselet, P., Comte, F.: Non-intrusive and exact global/local techniques for structural problems with local plasticity. Computational Mechanics 44(2), 233-245 (2009). DOI 10.1007/s00466-009-0372-9

[8] Gerasimov, T., Noii, N., Allix, O., De Lorenzis, L.: A non-intrusive global/local approach applied to phase-field modeling of brittle fracture. Advanced Modeling and Simulation in Engineering Sciences 5(1), 1-30 (2018). DOI 10.1186/s40323-018-0105-8. URL https://doi.org/10.1186/ 
S40323-018-0105-8

[9] Gosselet, P., Blanchard, M., Allix, O., Guguin, G.: Non-invasive global-local coupling as a Schwarz domain decomposition method: acceleration and generalization. Advanced Modeling and Simulation in Engineering Sciences 5(1) (2018). DOI 10.1186/s40323-018-0097-4

[10] Guguin, G., Allix, O., Gosselet, P., Guinard, S.: On the computation of plate assemblies using realistic 3D joint model: a non-intrusive approach. Advanced Modeling and Simulation in Engineering Sciences 3(1), 1-23 (2016). DOI 10.1186/s40323-016-0069-5

[11] Liu, Y.J., Sun, Q., Fan, X.L.: A non-intrusive global/local algorithm with non-matching interface: Derivation and numerical validation. Computer Methods in Applied Mechanics and Engineering 277(August), 81-103 (2014). DOI 10.1016/j.cma.2014.04.012. URL http://dx.doi.org/10. 1016/j.cma.2014.04.012

[12] Lorenzis, L.D.: Modeling in Engineering Using Innovative Numerical Methods for Solids and Fluids, CISM International Centre for Mechanical Sciences, vol. 599. Springer International Publishing, Cham (2020). DOI 10.1007/978-3-030-37518-8. URL http://link.springer.com/ $10.1007 / 978-3-030-37518-8$

[13] Passieux, J.c., Réthoré, J., Gravouil, A., Baietto, M.C.: Local / global non-intrusive crack propagation simulation using a multigrid X-FEM solver. Computational Mechanics 52(6), 1381-1393 (2013)

[14] Whitcomb, J.D.: Iterative global/local finite element analysis. Computers and Structures 40(4), 1027-1031 (1991). DOI 10.1016/0045-7949(91)90334-I 